Check for updates

Cite this: Phys. Chem. Chem. Phys., 2020, 22, 9775

Received 12th February 2020, Accepted 16th April 2020

DOI: $10.1039 / \mathrm{d} 0 \mathrm{cp} 00801 \mathrm{j}$

rsc.li/pccp

\section{Interaction of imidazolium-based lipids with phospholipid bilayer membranes of different complexity $\dagger$}

\author{
Steffen Bornemann, $\ddagger^{\mathrm{a}}$ Marius Herzog, $\ddagger^{\mathrm{a}}$ Lena Roling, ${ }^{\mathrm{b}}$ Tiffany O. Paulisch, ${ }^{\mathrm{b}}$ \\ Dörte Brandis, ${ }^{a}$ Simon Kriegler, ${ }^{a}$ Hans-Joachim Galla, iD c Frank Glorius iD *b and \\ Roland Winter (iD *a
}

\begin{abstract}
In recent years, alkylated imidazolium salts have been shown to affect lipid membranes and exhibit general cytotoxicity as well as significant anti-tumor activity. Here, we examined the interactions of a sterically demanding, biophysically unexplored imidazolium salt, 1,3-bis(2,6-diisopropylphenyl)-4,5-diundecylimidazolium bromide $\left(\mathbf{C}_{11} \mathrm{IPr}\right)$, on the physico-chemical properties of various model biomembrane systems. The results are compared with those for the smaller headgroup variant 1,3-dimethyl-4,5-diundecylimidazolium iodide $\left(\mathbf{C}_{11} \mathbf{I M e}\right)$. We studied the influence of these two lipid-based imidazolium salts at concentrations from 1 to about 10 mol\% on model biomembrane systems of different complexity, including anionic heterogeneous raft membranes which are closer to natural membranes. Fluorescence spectroscopic, DSC, surface potential and FTIR measurements were carried out to reveal changes in membrane thermotropic phase behavior, lipid conformational order, fluidity and headgroup charge. Complementary AFM and confocal fluorescence microscopy measurements allowed us to detect changes in the lateral organization and membrane morphology. Both lipidated imidazolium salts increase the membrane fluidity and lead to a deterioration of the lateral domain structure of the membrane, in particular for $\mathbf{C}_{11} \mathrm{IPr}$ owing to its bulkier headgroup. Moreover, partitioning of the lipidated imidazolium salts into the lipid vesicles leads to marked changes in lateral organization, curvature and morphology of the lipid vesicles at high concentrations, with $\mathbf{C}_{\mathbf{1 1}} \mathrm{IPr}$ having a more pronounced effect than $\mathrm{C}_{11} \mathrm{IMe}$. Hence, these compounds seem to be vastly suitable for biochemical and biotechnological engineering, with high potentials for antimicrobial activity, drug delivery and gene transfer.
\end{abstract}

\section{Introduction}

In 1991, the first free N-heterocyclic carbene (NHC) was isolated ${ }^{1}$ and from this time onwards the interest in NHCs has grown, especially in the fields of organometallic complexes, materials, drugs and catalysis. ${ }^{2-7}$ Initially, less attention was given to their corresponding imidazolium salts, which reveal a high potential to mimic lipid membrane properties with one or two lipid chains attached. These molecules belong to the class of ionic liquids (ILs), which generally serve as "designer solvents", and are used for numerous applications, including, for example, electrocatalysis,

\footnotetext{
${ }^{a}$ TU Dortmund University, Faculty of Chemistry and Chemical Biology, Physical Chemistry I - Biophysical Chemistry, Otto Hahn Str. 4a, D-44221 Dortmund, Germany. E-mail: roland.winter@tu-dortmund.de

${ }^{b}$ University of Münster, Institute of Organic Chemistry, Corrensstraße 40, 48149 Münster, Germany.E-mail: glorius@uni-muenster.de

${ }^{c}$ University of Münster, Institute of Biochemistry, Wilhelm Klemm Str. 2, 48149 Münster, Germany. E-mail: gallah@uni-muenster.de

$\dagger$ Electronic supplementary information (ESI) available. See DOI: 10.1039/d0cp00801j

\$ These authors are co-first authors.
}

extraction processes and bio-refineries, or as electrolytes for power sources and solar cells. ${ }^{8,9}$ Today, imidazolium-based molecules are also discussed as antimicrobial and antifungal drugs for clinical use and they have become an attractive target against cancers. ${ }^{10,11}$ Imidazolium salts with bulky, aromatic and long alkyl chain substitutions have shown the highest effectiveness against various tumor cell lines. ${ }^{10}$ A clear advantage of imidazolium salts is the ease of making structural changes that can modify and adjust the desired biophysical properties such as lipophilicity, solubility and amphiphilicity to maintain high antitumor activities with low cytotoxic behavior towards healthy cells. ${ }^{10-12}$ A strong correlation was observed between the hydrophobic strength of imidazoliumbased ionic liquids and cell cycle as well as cell death of mammalian cells, caused by enhanced dynamics, disorder and changes in the self-assembled structure of the cell membranes. ${ }^{12}$ Furthermore, the $\mathrm{N}$-substituted imidazole ring can be found in copious bioactive molecules of the human metabolism and in natural products. Their amphiphilic and highly polar heterocycle is prone for electrostatic interactions with different biomacromolecular systems, especially biological membranes. Recent studies have revealed a remarkable 
membrane activity of imidazolium salts. ${ }^{13-16}$ Alkylated imidazolium compounds demonstrate detergent-like properties due to their cationic headgroup and adjustable alkyl chain length. ${ }^{17-20}$ Encouraged by using molecular hybridization as strategy for developing medicinal agents, the Glorius group designed and synthesized various backbone alkylated imidazolium lipids, which differ in the length of their two hydrophobic alkyl chains at the 4- and 5-position of the imidazole ring and their cationic headgroup structure. ${ }^{21-23}$ Owing to their structural similarity to natural phospholipids like dipalmitoylphosphatidylcholine (DPPC), these molecules have the possibility to mimic physio-chemical properties of natural lipids. It was shown that these artificial lipids own considerable antitumor activity and cellular toxicity. ${ }^{21,22}$ Their general cytotoxicity is strongly dependent on the chain length of the alkyl chains at the 4- and 5 -position of the imidazole ring. A longer alky chain $\left(\mathrm{C}_{15}\right)$ has been shown to have the lowest and a short alkyl chain $\left(\mathrm{C}_{7}\right)$ the most potent cellular toxicity. ${ }^{21,22}$ The surface activity is much higher for longer alkyl chains, which indicates that the biological action strongly depends on their biophysical properties. Studies on the interaction with phospholipid membranes revealed that $\mathbf{C}_{\mathbf{1 5}}$ IMe readily inserts into the lipid membrane. $\mathbf{C}_{\mathbf{1 1}}$ IMe has a lytic and destabilizing effect, whereas $\mathbf{C}_{7} \mathbf{I M e}$ is almost non-lytic due to its short alkyl chainlength. ${ }^{21}$ Another study focused on the effects of different headgroup substitutions. It was pointed out that small and hydrophilic substituents cause a rigidification of the DPPC membrane, while bulky and hydrophobic substituents fluidize the DPPC membrane. ${ }^{23}$ In general, ILs have demonstrated a disordering effect and reduction of the elasticity leading to perturbations of self-assembled structures of cellular membranes. ${ }^{24}$

In this work, we studied one so far biophysically uncharacterized imidazolium-based lipid, the $\mathbf{C}_{\mathbf{1 1}} \mathbf{I P r}$, which was used to explore the effect of a bulkier and more hydrophobic headgroup on various lipid membranes in comparison to that of $\mathbf{C}_{\mathbf{1 1}}$ IMe, which has in part been described before ${ }^{21,22,25,26}$ (for the structures, see Fig. 1). Generally, imidazolium salts with a hydrophilic and polar headgroup are incorporated more easily into model membranes, whereas bulky and hydrophobic headgroups cause vacancies and destabilization of lipid bilayers. ${ }^{23}$ Moreover, the experiments were carried out using model biomembranes of different complexity. We started with a onecomponent lipid membrane consisting of DPPC and increased the membrane complexity using DPPC:DOPC: cholesterol $(2: 1: 1)$ as neutral heterogeneous bilayer and further a five component anionic raft mixture of DPPC:DPPG:DOPC:DOPG: cholesterol $(45: 5: 20: 5: 25)$, which mimics more the natural lipid membrane since the typical physiological concentration of anionic lipids in the plasma membrane amounts to about $10 \mathrm{~mol} \% .^{27,28}$ Furthermore, negatively charged lipids have shown on the one hand a disordering effect on acyl chains in the membrane and, in combination with ILs, displayed a vesicle-stabilizing character. $^{29}$ The more-component membrane systems containing cholesterol show coexistence of a raft-like liquid-ordered $\left(l_{o}\right)$ phase and a more fluid, liquid-disordered $\left(l_{d}\right)$ phase at ambient temperature. ${ }^{30,31}$ The existence of domains of different composition and fluidity seems to be important for various membrane-associated processes, including signaling, exo- and endocytosis and virus uptake. ${ }^{31-34}$

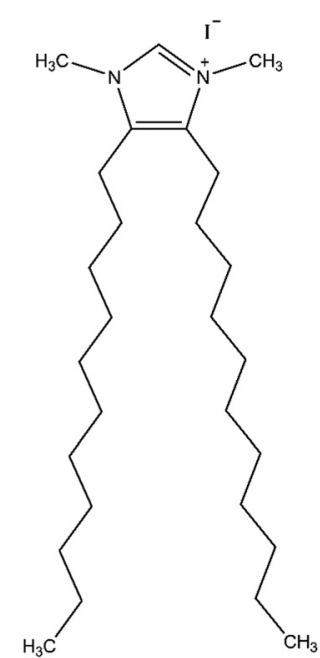

$\mathrm{C}_{11}$ IMe

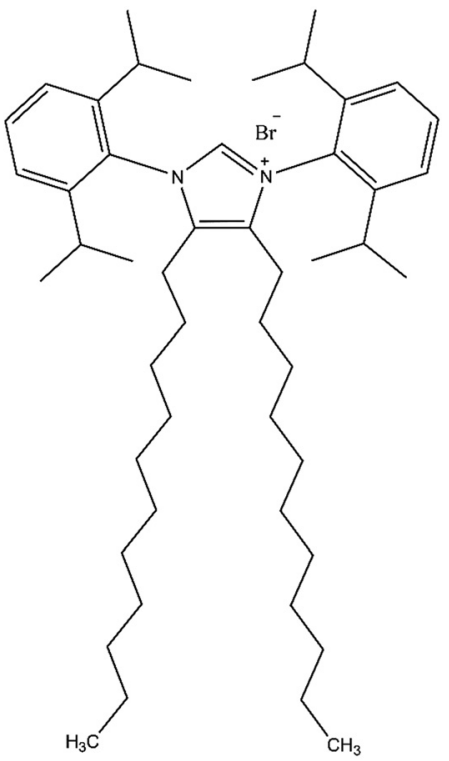

$\mathrm{C}_{11} \mathrm{IPr}$
Fig. 1 Structures of the imidazolium-based lipid salts investigated in this study.

On the other hand, the one-component DPPC membrane displays single lamellar phases with two major phase transitions with increasing temperature, a gel-to-gel pretransition $\left(\mathrm{L}_{\beta^{\prime}} / \mathrm{P}_{\beta^{\prime}}\right)$ at $\sim 35{ }^{\circ} \mathrm{C}$, and a gel-to-fluid main transition $\left(\mathrm{P}_{\beta^{\prime}} / \mathrm{L}_{\alpha}\right)$ at $\sim 41{ }^{\circ} \mathrm{C}^{35-39}$

\section{Materials and methods}

\subsection{Sample preparation}

The phospholipids 1,2-dipalmitoyl-sn-glycero-3-phosphocholine (DPPC), 1,2-dipalmitoyl-sn-glycero-3-phospho-(1'-rac-glycerol) sodium salt (DPPG), 1,2-dioleoyl-sn-glycero-3-phosphocholine (DOPC), 1,2-dioleoyl-sn-glycero-3-phospho-(1'-rac-glycerol) sodium salt (DOPG) and the cholesterol were purchased from Avanti Polar Lipids (Alabaster, AL) with purities of $>99 \%$ and were used without further purification. The fluorescently labeled $N$-(lissamine ${ }^{\text {TM }}$ rhodamin B sulfonyl)-1,2-dihexadecanoyl-sn-glycero-3-phosphoethanolamine ( $N$-rhodamine(Rh)-DHPE) for the microscopy investigations was purchased from Invitrogen (Carlsbad, California, USA). The fluorescence probe Laurdan (6-dodecanoyl- $N, N$-dimethyl2-naphthylamine) was obtained from Sigma-Aldrich (St. Louis, USA). All other solvents and chemicals were purchased from SigmaAldrich (St. Louis, USA) and Merck (Darmstadt, Germany).

The different lipid mixtures were prepared by mixing stock solutions with a concentration of $10 \mathrm{mg} \mathrm{mL} \mathrm{m}^{-1}$ of the pure components in chloroform or chloroform/methanol. The lipid mixtures prepared contained (a) pure DPPC, (b) $2: 1: 1 \mathrm{~mol} \%$ DPPC : DOPC : cholesterol and (c) $20: 5: 45: 5: 25$ mol\% DOPC: DOPG : DPPC:DPPG : cholesterol. The imidazolium salts were always added before vesicle preparation. The imidazolium salts containing lipid systems were mixed in the given molar ratios. The concentration of DPPC was reduced by the amount by which 
the concentration of the imidazolium salts was increased. For the fluorescence spectroscopy and microscopy measurements, $10 \mu \mathrm{L}$ of a $1 \mathrm{mM}$ Laurdan solution and $0.1 \mathrm{~mol} \% \mathrm{~N}$-rhodamine-DHPE were added to the lipid mixtures, respectively. The preparation of multilamellar, giant and large vesicles (MLVs, GUVs, LUVs) followed protocols as described in the literature. ${ }^{36,38,39}$ All measurements were carried out at least three times using independently prepared samples.

\section{$2.2 \quad \mathrm{C}_{11} \mathrm{IMe}$ and $\mathrm{C}_{11} \mathrm{IPr}$ imidazolium based lipid salts}

In this study, we used two different imidazolium molecules. The first one, the 1,3-dimethyl-4,5-diundecylimidazolium iodide, is used as benchmark, since it has already been studied to a certain extent ${ }^{40}$ and is referred to as $\mathbf{C}_{\mathbf{1 1}} \mathbf{I M e}$ in the following. The second molecule is based on a design with a modified headgroup, 1,3bis(2,6-diisopropylphenyl)-4,5-diundecylimidazolium bromide, and referred to as $\mathbf{C}_{\mathbf{1 1}}$ IPr in the following. Its interaction with lipid bilayer membranes is in the focus of this study. As depicted in Fig. 1, the headgroup of $\mathbf{C}_{\mathbf{1 1}}$ IPr is sterically more demanding. The methods used for the studies on $\mathbf{C}_{\mathbf{1 1}} \mathbf{I M e}$ and $\mathbf{C}_{\mathbf{1 1}}$ IPr were selected to reveal their interaction (changes in phase behavior, lipid order parameter, charge density, morphology, etc.) with different types of membranes.

\subsection{Fourier-transform infrared (FTIR) spectroscopy}

The MLVs were prepared at a total lipid concentration of $10 \mathrm{wt} \%$ in $10 \mathrm{mM}$ HEPES buffer in $\mathrm{D}_{2} \mathrm{O}$ at a $\mathrm{pH}$ of 7.4. For the FTIR experiments, five concentrations of $\mathbf{C}_{\mathbf{1 1}} \mathrm{IMe}$ and $\mathbf{C}_{\mathbf{1 1}} \mathrm{IPr}$ from $0 \mathrm{~mol} \%$ up to $15 \mathrm{~mol} \%$ for the pure DPPC, and three concentrations from $0 \mathrm{~mol} \%$ to $10 \mathrm{~mol} \%$ for the $2: 1: 1 \mathrm{~mol} \%$ DPPC: DOPC:cholesterol as well as for the 20:5:45:5:25 mol\% DOPC:DOPG:DPPC:DPPG : cholesterol mixture were utilized. The temperature-dependent FTIR spectra were recorded using a sample cell consisting of two IR-transparent $\mathrm{CaF}_{2}$ windows (Korth Kristalle GmbH, Germany) separated by a film gasket of $50 \mu \mathrm{m}$ thickness, providing space for $20 \mu \mathrm{L}$ of sample solution. The resolution of the recorded spectra was $2 \mathrm{~cm}^{-1}$ consisting of 128 averaged separate scans on a Bruker Vertex-70 FT-IR spectrometer (Billerica, Massachusetts, USA). Contributions of $\mathrm{CO}_{2}$ or $\mathrm{H}_{2} \mathrm{O}$ from the surrounding gas phase were excluded by purging the measurement chamber with carbon dioxide-free, dried air for at least fifteen minutes before each measurement. The temperature was varied by use of an external water thermostat from Julabo (Seelbach, Germany) controlled by the Julabo Easy Temp software and a programmed temperature ramp. Before each measurement, the temperature of the sample was equilibrated for $15 \mathrm{~min}$ and the sample temperature was logged every $3 \mathrm{~s}$ by an external Pt-100 thermometer attached to the measurement chamber. The measured temperature ranges were from 25 to $55{ }^{\circ} \mathrm{C}$ for DPPC and from 10 to $80{ }^{\circ} \mathrm{C}$ for DPPC: DOPC: cholesterol and DOPC:DOPG : DPPC : DPPG : cholesterol, respectively.

\subsection{Differential scanning calorimetry (DSC)}

DSC measurements were carried out for the pure DPPC system upon addition of several concentrations from $0 \mathrm{~mol} \%$ to $15 \mathrm{~mol} \%$ for $\mathbf{C}_{\mathbf{1 1}} \mathbf{I M e}$ and from $0 \mathrm{~mol} \%$ to $50 \mathrm{~mol} \%$ for $\mathbf{C}_{\mathbf{1 1}} \mathbf{I P r}$.
Sample preparation followed the preparation described for the MLVs as mentioned above. The DSC measurements were executed on a Q20 Differential Scanning Calorimeter (TA Instruments, New Castle, USA) using sample cells consisting of Tzero ${ }^{\circledR}$ pans, purchased from TA Instruments (New Castle, USA), which were filled with $20 \mu \mathrm{L}$ of sample solution, whereas the reference cell was filled with $20 \mu \mathrm{L}$ buffer solution. Before each heating scan with a heat rate of $1.5{ }^{\circ} \mathrm{C} \mathrm{min}^{-1}$, the sample solution was equilibrated at the chosen start temperature for $5 \mathrm{~min}$.

\subsection{Fluorescence spectroscopy (FS)}

Fluorescence spectroscopy using the fluorescent probe Laurdan was used to obtain information about the temperature-induced changes of the lateral order of the model membranes and the membrane fluidity. The measured temperature range was from 10 to $80{ }^{\circ} \mathrm{C}$. The sample preparation proceeded similar to the sample preparation for the FTIR and DSC experiments. The total lipid concentration was $10 \mathrm{mg} \mathrm{mL}^{-1}$ and a 6-dodecanoyl$N, N$-dimethyl-2-naphthylamine (Laurdan) solution was added at a ratio of 1 mol lipid: 0.000735 mol Laurdan. The imidazolium salt concentrations chosen were $5 \mathrm{~mol} \%$ and $10 \mathrm{~mol} \%$ for $\mathbf{C}_{\mathbf{1 1}}$ IMe and $\mathbf{C}_{\mathbf{1 1}} \mathrm{IPr}$ (only $10 \mathrm{~mol} \%$ for pure DPPC experiments). Investigations of $\mathbf{C}_{\mathbf{1 1}}$ IMe and $\mathbf{C}_{\mathbf{1 1}}$ IPr were carried out on pure DPPC as well as on the three- and the five-component lipid mixture. The measurements were carried out on a K2 multifrequency phase modulation fluorometer (ISS Inc., Champaign, IL). The fluorophore was excited at a wavelength of $390 \mathrm{~nm}$ and the emission intensity was collected in a range of $420 \mathrm{~nm}$ to $520 \mathrm{~nm}$. Quantification of the spectral changes was achieved by use of the generalized polarization $(G P)$ function, which is defined as $G P=\left(I_{\mathrm{B}}-I_{\mathrm{R}}\right) /\left(I_{\mathrm{B}}+I_{\mathrm{R}}\right)$, with $I_{\mathrm{R}}$ referring to the fluorescence intensity at $490 \mathrm{~nm}$ and $I_{\mathrm{B}}$ referring to the fluorescence intensity at $440 \mathrm{~nm}$. High emission at $440 \mathrm{~nm}$ indicates an ordered gel phase, whereas an emission maximum at $490 \mathrm{~nm}$ is characteristic for a fluid (liquid-crystalline) phase. Hence, increasing GP-values indicate an increasing lateral order of the lipid acyl chains, while decreasing $G P$-values indicate increasing disorder and increasing fluidity. For all systems measured, the photon count was averaged over a time span of $1 \mathrm{~s}$. Temperature control was achieved using a circulating water bath (Julabo, Seelbach, Germany) attached to the measurement cell and monitoring the temperature with a thermocouple inserted into the cell.

\subsection{Fluorescence microscopy (FM)}

Fluorescence microscopy measurements have been performed on the pure DPPC and heterogeneous three- and anionic fivecomponent lipid mixtures. The necessary amount of stock solutions of the lipids were mixed with chloroform to obtain $100 \mu \mathrm{L}$ solution with a concentration of $1 \mathrm{mg} \mathrm{mL} \mathrm{m}^{-1}$ lipid in the desired ratio (DOPC:DOPG:DPPC:DPPG : cholesterol 20:5:45:5:25). The imidazolium salts at the corresponding concentrations were added before vesicle preparation. About $3 \mu \mathrm{L}$ of the $0.1 \mathrm{mM}$ concentrated fluorophore $N$-Rh-DHPE were added to this solution to obtain a final molar ratio of lipid to dye of 500:1 (0.1 mol\%). GUVs were obtained by spin coating the lipid solutions onto pre-cleaned optically transparent and electrically 
conductive indium tin oxide (ITO) coated glass slides (SPI Supplies, West Chester, USA) at $800 \mathrm{rpm}$ for $60 \mathrm{~s}$ and removing the remaining solvent by evaporation by freeze-drying the covered glass slides under vacuum for at least $2 \mathrm{~h}$. Afterwards, the glass slides were used to prepare GUVs via electroformation ${ }^{41,42}$ in a type RC-21B formation chamber (Warner Instruments, Hamden, USA) at $75{ }^{\circ} \mathrm{C}$. GUVs consisting of DPPC and the three component lipid mixtures were obtained by using an alternating electric field of $2 \mathrm{~V}$ at a high frequency of $500 \mathrm{~Hz}$ applied to the ITO slides by using a function generator TG315 (Thurlby Thandar Instruments, Huntingdon, UK). ${ }^{43}$ GUVs of the anionic five component mixture were prepared by using an alternating electric field of $0.14 \mathrm{~V}$ for $5 \mathrm{~min}, 1.25 \mathrm{~V}$ (20 $\mathrm{min}$ ) and $3.5 \mathrm{~V}$ (90 min) and a constant low frequency of $10 \mathrm{~Hz}$. After completion, the temperature was reduced to room temperature steadily by $1{ }^{\circ} \mathrm{C}$ per minute. Temperature-dependent measurements were carried out in the ITO cell by heating with a circulating water bath (Julabo, Seelbach, Germany). The images were recorded by use of a confocal laser scanning microscope (Biorad MRC 1042, Zeiss, Germany), which was coupled via a side-port to an inverted microscope (Nikon, Eclipse TE-300 DV, infinity corrected optics). Fluorescence at excitation wavelengths of $488 \mathrm{~nm}, 561 \mathrm{~nm}$ and $648 \mathrm{~nm}$ was enabled by a laser combiner (Oxxius Simply Light, L4Cc-CSB130, Lannion, France). Image acquisition was controlled by the software LaserSharp2000 (Zeiss, Germany). The images were taken using the oil-supported objective lens Typ CFI Plan Apo $\lambda$ $100 \times$ Oil, NA 1.45 WD 0.13 (Nikon, Tokyo, Japan). For all systems and temperatures investigated, images were taken in various areas of each sample.

\subsection{Dynamic light scattering (DLS)}

For obtaining size information of the aqueous suspensions of pure DPPC and the five-component lipid vesicles and determination of the zeta potential of the electrical double layer lipid interface relative to the bulk fluid, DLS and zeta potential measurements were carried out for the $\mathbf{C}_{\mathbf{1 1}}$ IMe and the $\mathbf{C}_{\mathbf{1 1}}$ IPr systems. Unilamellar vesicles with a diameter of $100 \mathrm{~nm}$ and the required composition were prepared as described for the FTIR or DSC experiments above. The total lipid concentration in the aliquots was $10 \mathrm{mM}$ each. Further, the aliquots were extruded 21 times through a polycarbonate filter of $100 \mathrm{~nm}$ pore size (Nuclepore ${ }^{\circledR}$ Polycarbonate Track-Etch $^{\mathrm{TM}}$, Whatman GmbH, Dassel, Germany) to obtain a homogeneous size distribution. The measurements were performed on a ZetaSizer Nano ZS, using disposable folded capillary cells DTS 1070 (Malvern Instruments, Malvern, UK). Measurements of particle size and surface potential were carried out at $25{ }^{\circ} \mathrm{C}$. For all systems investigated, the results were averaged over three independent measurements, each consisting of at least 15 single scans.

\subsection{Atomic force microscopy (AFM)}

The initial sample preparation was similar to the FTIR-spectroscopy samples. Rehydration of the dried lipid mixture was carried out with buffer consisting of $20 \mathrm{mM}$ TRIS (tris(hydroxymethyl)aminomethane) and $5 \mathrm{mM} \mathrm{MgCl}_{2}$ with a $\mathrm{pH}$ of 7.4 , gaining a total lipid concentration of $1.94 \mathrm{mg} \mathrm{mL}^{-1}$. Preparation instructions can be found in detail in ref. 44 . The multilamellar vesicles were extruded through a $100 \mathrm{~nm}$ pore filter and deposited onto a mica substrate surface for vesicle fusion for $2 \mathrm{~h}$ at $80{ }^{\circ} \mathrm{C}$. After multilayer formation, the surface was rinsed several times with buffer solution to remove unfused vesicles. Measurements were taken on a MultiMode scanning probe microscope, controlled by a NanoScope IIIa controller (Digital Instruments, Santa Barbara, CA) and a J-Scanner. Images were taken using tips with a nominal force constant of $0.35 \mathrm{~N} \mathrm{~m}^{-1}$ in liquid via tapping mode in a fluid cell (MTFML, Veeco, Mannheim, Germany) at a scan frequency of 1.0 $\mathrm{Hz}$ and at least at three different positions on each membrane for evaluation.

\subsection{CMC determination}

For determination of the critical micelle concentration $(\mathrm{cmc})$, a $0.1 \mathrm{mM}$ stock solution of $\mathbf{C}_{\mathbf{1 1}} \mathbf{I P r}$ was prepared in chloroform and the surface pressure, $\pi$, was measured four times at $20{ }^{\circ} \mathrm{C}$ with a Surface Pressure Sensor Type PS4 (NIMA Technology Ltd, Coventry, England). A detailed description of the measuring procedure and cmc determination can be found in ref. 23 and 25 .

\section{Results and discussion}

\subsection{CMC determination}

Characterization of the surface activity of the new imidazolium salt was carried out by determining the critical micelle concentration, cmc, of $\mathbf{C}_{\mathbf{1 1}}$ IPr by use of the film balance technique. Fig. 2 shows the resulting isotherm at room temperature of the surface tension plotted as a function of $\mathbf{C}_{\mathbf{1 1}} \mathbf{I P r}$ concentration.

From the fit shown in Fig. 2, the cmc was determined as $0.7 \pm 0.08 \mu \mathrm{M}$, which is in the same order of magnitude as the cmc-values of the structurally similar compounds $\mathbf{C}_{\mathbf{1 1}} \mathbf{I B n}(1,3-$ dibenzyl-4,5-diundecylimidazolium bromide) and $\mathbf{C}_{\mathbf{1 1}}$ IMe (Table 1). The cmc of $\mathbf{C}_{\mathbf{1 1}} \mathbf{I P r}$ is slightly smaller than the cmc of $\mathbf{C}_{\mathbf{1 1}} \mathbf{I B n}$, probably due to the additional four isopropyl-residues in the headgroup region. The measurements with the lipid components

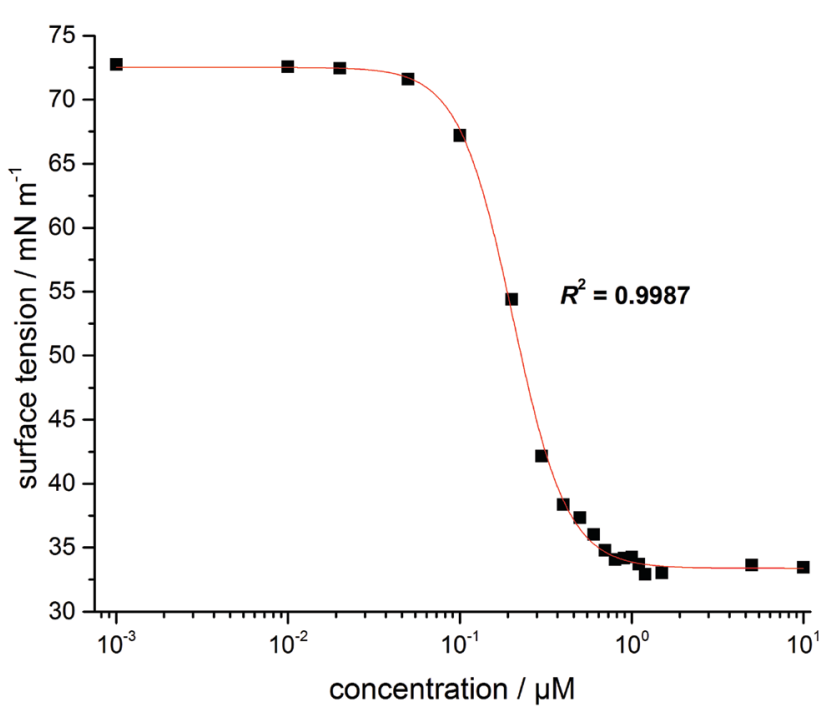

Fig. 2 Plot of the isotherm of the surface tension against the concentration for $\mathrm{C}_{11} \mathrm{IPr}$. 
Table 1 CMC values of $\mathbf{C}_{11} \mathrm{IPr}$ in comparison to the $\mathrm{cmc}$ values of structurally similar imidazolium salts

\begin{tabular}{ll}
\hline Substrate & $\mathrm{cmc} / \mu \mathrm{M}$ \\
\hline $\mathbf{C}_{\mathbf{1 1}} \mathrm{IMe}^{21,22}$ & $5-10$ \\
$\mathbf{C}_{\mathbf{1 1}} \mathrm{IBn}^{21}$ & $2.0-5.0$ \\
$\mathbf{C}_{\mathbf{1 1}} \mathrm{IPr}$ (this work) & $0.7 \pm 0.08$
\end{tabular}

described below are hence carried out at concentrations far beyond the cmc of both imidazolium salts.

\subsection{Temperature-dependent FTIR measurements}

To gain information on the conformational order of the lipid chains in the model membranes studied, we monitored the alterations in the vibrational modes in the IR-spectra in a spectral range between $2800 \mathrm{~cm}^{-1}$ and $3100 \mathrm{~cm}^{-1} \cdot{ }^{36,44}$ As indicator for the gel-to-fluid membrane phase transitions, the asymmetric
$\mathrm{CH}_{2}$-vibrational mode between $2916 \mathrm{~cm}^{-1}$ and $2925 \mathrm{~cm}^{-1}$ was analyzed. Due to temperature-induced changes in the transgauche ratio of the acyl chains, a distinct change in the maximum position of this specific band was observed. ${ }^{37,45}$ Hence, the shift in the maximum wavenumber, $\Delta \tilde{v}$, of this band can be used as a marker for the fluidity and change in phase behavior of the membrane.

In the pure DPPC membrane, the insertion of $\mathbf{C}_{\mathbf{1 1}} \mathbf{I M e}$ results in a decrease of the main gel-to-fluid phase transition temperature, $T_{\mathrm{m}}$, indicating a fluidizing effect and stabilization of the fluid phase against decreasing temperatures. The maximum effect occurs already at about $7.5 \mathrm{~mol} \% \mathbf{C}_{\mathbf{1 1}} \mathbf{I M e}$, resulting in a decreased $T_{\mathrm{m}}$ of about $3{ }^{\circ} \mathrm{C}$ compared to the pure lipid bilayer membrane. Further increase of the $\mathbf{C}_{\mathbf{1 1}} \mathbf{I M e}$ concentration up to $15 \mathrm{~mol} \%$ does not lead to a further significant decrease of the phase transition temperature. Insertion of $\mathbf{C}_{\mathbf{1 1}} \mathbf{I P r}$ into the pure DPPC system shows much more pronounced effects. Whereas at a
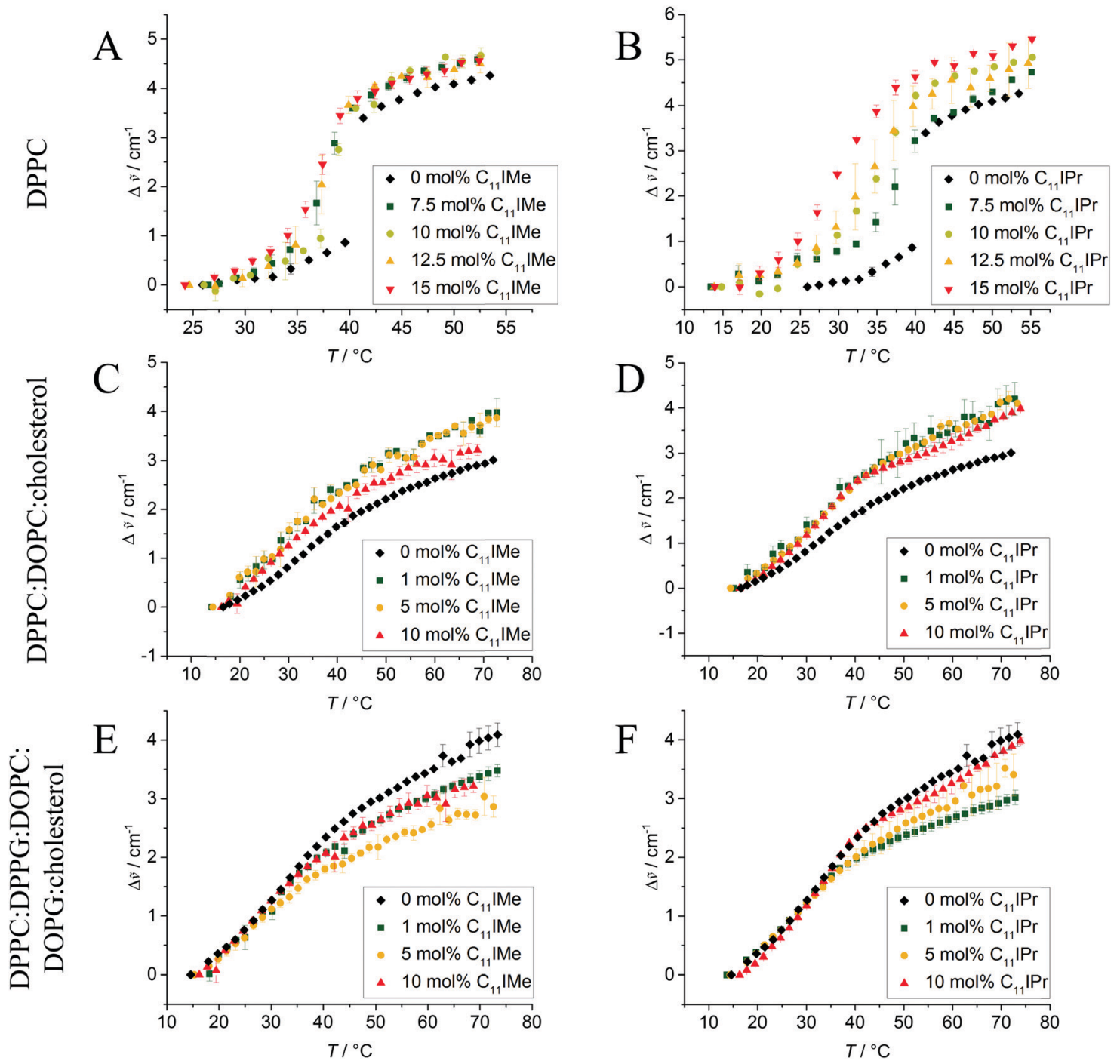

Fig. 3 Wavenumber shifts, $\Delta \tilde{\nu}$, of the $\mathrm{CH}_{2}$ vibrational band of (A and B) pure DPPC, (C and D) 2:1:1 DPPC:DOPC: cholesterol and (E and F) (45:5:20:5:25) DPPC: DPPG : DOPC: DOPG: cholesterol without and with $\mathrm{C}_{11} \mathbf{I} \mathbf{M e}(\mathrm{A}, \mathrm{C}$ and $\mathrm{E})$ and $\mathrm{C}_{11} \mathbf{I P r}(\mathrm{B}, \mathrm{D}$ and F). The heterogeneous lipid systems exhibit extended phase coexistence regions, while the pure DPPC membrane undergoes a relatively sharp gel-to-fluid phase transition. All transitions are significantly affected by the insertion of the imidazolium salts. 
$\mathbf{C}_{11}$ IPr mole fraction of $7.5 \mathrm{~mol} \%$ a decrease in $T_{\mathrm{m}}$ of about $2{ }^{\circ} \mathrm{C}$ is detected only, a further increase of the $\mathbf{C}_{\mathbf{1 1}} \mathbf{I P r}$ concentration up to $15 \mathrm{~mol} \%$ leads to a transition temperature decrease of $10{ }^{\circ} \mathrm{C}$. This indicates a marked disturbance of the packing of lipid molecules in the lipid bilayer induced by both imidazolium salts, with $\mathbf{C}_{\mathbf{1 1}} \mathbf{I P r}$ imposing a more pronounced effect. The weaker intermolecular interactions between the acyl chains of the lipid membrane can be explained by the shorter chain length of the imidazolium salts, causing hydrophobic mismatch. The significant difference between the $\mathbf{C}_{\mathbf{1 1}} \mathbf{I M e}$ and the $\mathbf{C}_{\mathbf{1 1}} \mathbf{I P r}$ induced effects most likely arises as a result of the much larger membrane disturbance imposed by the bulkier headgroup of the $\mathbf{C}_{\mathbf{1 1}} \mathbf{I P r}$.

The FTIR data of the effect of $\mathbf{C}_{\mathbf{1 1}}$ IMe and $\mathbf{C}_{\mathbf{1 1}}$ IPr on the heterogeneous membranes 2:1:1 DPPC:DOPC:cholesterol and $45: 5: 20: 5: 25$ DPPC:DPPG:DOPC:DOPG : cholesterol are shown in Fig. 3. Owing to their more complex composition, broad transition regions are observed in these cases. The multicomponent nature of these lipid systems results in broad phase coexistence regions of liquid-ordered and liquid-disordered domains, hence lack a sharp gel-to-fluid phase transition. As expected, both imidazolium salts have an effect on the order of these heterogeneous membranes as well. Insertion of $\mathbf{C}_{\mathbf{1 1}} \mathbf{I M e}$ and $\mathbf{C}_{\mathbf{1 1}}$ IPr into the neutral three component system increases the wavenumber of the $\mathrm{CH}_{2}$-vibrational band in the whole temperature range investigated, indicating a higher amount of disorder (e.g., gauche conformers) and hence a fluidizing effect. Insertion into the anionic five component system displays a similar scenario. To shed more light on the effects occurring in these heterogeneous membranes, complementary fluorescence spectroscopy, fluorescence microscopy and atomic force microcopy experiments have been carried out on these systems, which are described below.

\subsection{Differential scanning calorimetry (DSC) measurements}

To yield additional thermodynamic information on the transition properties of the membrane systems in the absence and presence of the inserted imidazolium salts, we performed differential scanning calorimetry measurements. Owing to the broad coexistence regions of the more-component lipid mixtures, the DSC scans are very broad and featureless and hence do no provide novel information. Hence, changes in transition temperatures are barely visible by this method. The pure DPPC system, however, shows distinct effects upon addition of the imidazolium salts on the transition behavior. As shown in Fig. 4, the main phase transition temperatures, $T_{\mathrm{m}}$, decreases as a result of the insertion of the imidazolium salts, in accordance with the FTIR results. The gel-to-gel pretransition temperature, $T_{\mathrm{p}}$, of the $\mathrm{DPPC} / \mathbf{C}_{\mathbf{1 1}} \mathbf{I M e}$ system also decreases with rising $\mathbf{C}_{\mathbf{1 1}} \mathbf{I M e}$ fraction and disappears at concentrations beyond $5 \mathrm{~mol} \% \mathbf{C}_{\mathbf{1 1}} \mathbf{I M e}$.

Fig. 5A reveals that the insertion of $\mathbf{C}_{\mathbf{1 1}}$ IMe leads to the disappearance of the $\mathrm{L}_{\beta^{\prime}}$-to- $\mathrm{P}_{\beta^{\prime}}$ gel-to-gel pretransition peak of the pure DPPC membrane at a mole fraction of $5.0 \mathrm{~mol} \%$, most likely as insertion of $\mathbf{C}_{\mathbf{1 1}} \mathbf{I M e}$ disturbs the efficient packing of neighboring lipids needed to establish the ripple gel phase $\left(\mathrm{P}_{\beta}{ }^{\prime}\right)$. Further increase of $\mathbf{C}_{\mathbf{1 1}} \mathrm{IMe}$ leads to a peak broadening, indicating formation of phase coexistence regions at high imidazolium salt concentrations. The insertion of $\mathbf{C}_{\mathbf{1 1}}$ IPr into the membrane shows

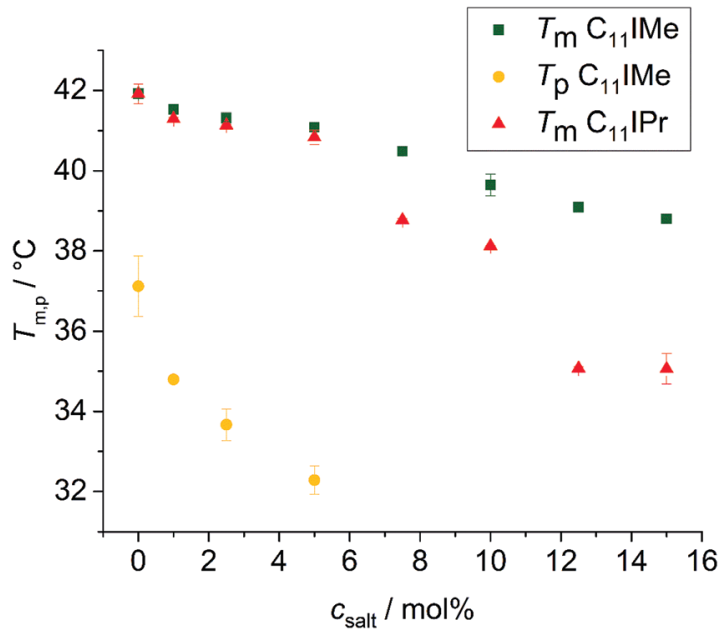

Fig. 4 Decrease of the main phase transition temperatures $T_{m}$, and the pretransition temperature, $T_{p}$, of the DPPC: $\mathbf{C}_{11} I M e$ and DPPC: $\boldsymbol{C}_{11} I \mathrm{Pr}$ mixtures at different imidazolium salt concentrations. The pretransition of the former disappears at $5 \mathrm{~mol} \%$ while the pretransition of the DPPC: $\mathrm{C}_{11} \mathrm{IPr}$ mixture is absent for all $\mathrm{C}_{11} \mathrm{IPr}$ salt concentrations.

even more pronounced effects on the lipid's phase behavior. A fraction of already 1 mol\% $\mathbf{C}_{\mathbf{1 1}} \mathbf{I P r}$ is sufficient to abolish the pretransition, resulting in a pronounced peak broadening with rising $\mathbf{C}_{\mathbf{1 1}} \mathrm{IPr}$ concentration, as can be seen in Fig. 5B. A detailed view of the broadened peaks is depicted in Fig. 5C, which shows the appearance of distinct, separate peaks at high fractions of $12.5 \mathrm{~mol} \%$ and $15 \mathrm{~mol} \% \mathbf{C}_{\mathbf{1 1}} \mathbf{I M e}$. At very high fractions of $25 \mathrm{~mol} \%$ and $50 \mathrm{~mol} \% \mathbf{C}_{\mathbf{1 1}} \mathrm{IPr}$, additional distinct DSC peaks are visible, indicating phase segregation into various distinct lipid bilayer domains or micellar structures. To gain more information on the lateral membrane organization under such conditions, such as the ability of $\mathbf{C}_{\mathbf{1 1}} \mathbf{I P r}$ to build mesoscopic structures on its own, additional experiments were carried out on neat $\mathbf{C}_{\mathbf{1 1}}$ IPr solutions. We observed that the compound is not able to build mesoscopic structures that undergo phase transitions with temperature. This indicates that the separated DSC peaks observed at mole fractions of $25 \mathrm{~mol} \%$ and $50 \mathrm{~mol} \% \mathbf{C}_{\mathbf{1 1}} \mathbf{I P r}$ in DPPC vesicles do not result from pure $\mathbf{C}_{\mathbf{1 1}} \mathbf{I P r}$ bilayer or lipid micellar structures, but rather from segregated domains or DPPCstabilized, mixed micellar structures. High concentrations of $\mathbf{C}_{\mathbf{1 1}}$ IMe have already been studied. The DSC data showed full disappearance of the phase transition at mole fractions beyond 60 mol\% $\mathbf{C}_{\mathbf{1 1}} \mathrm{IMe}^{26}$

\subsection{Fluorescence spectroscopy measurements}

Information about changes in the lipid order parameter were obtained by use of fluorescence spectroscopy measurements with Laurdan as fluorescent probe. We investigated the impact of both lipid-like imidazolium salts on pure DPPC bilayers, the three-component membrane consisting of DPPC:DOPC: cholesterol and the anionic five-component raft mixture. For each measurement, the $G P$-values were calculated from averaged intensities at $440 \mathrm{~nm}$ and $490 \mathrm{~nm}$ and plotted against the temperature. The results are depicted in Fig. 6. 
A

$\mathrm{C}$
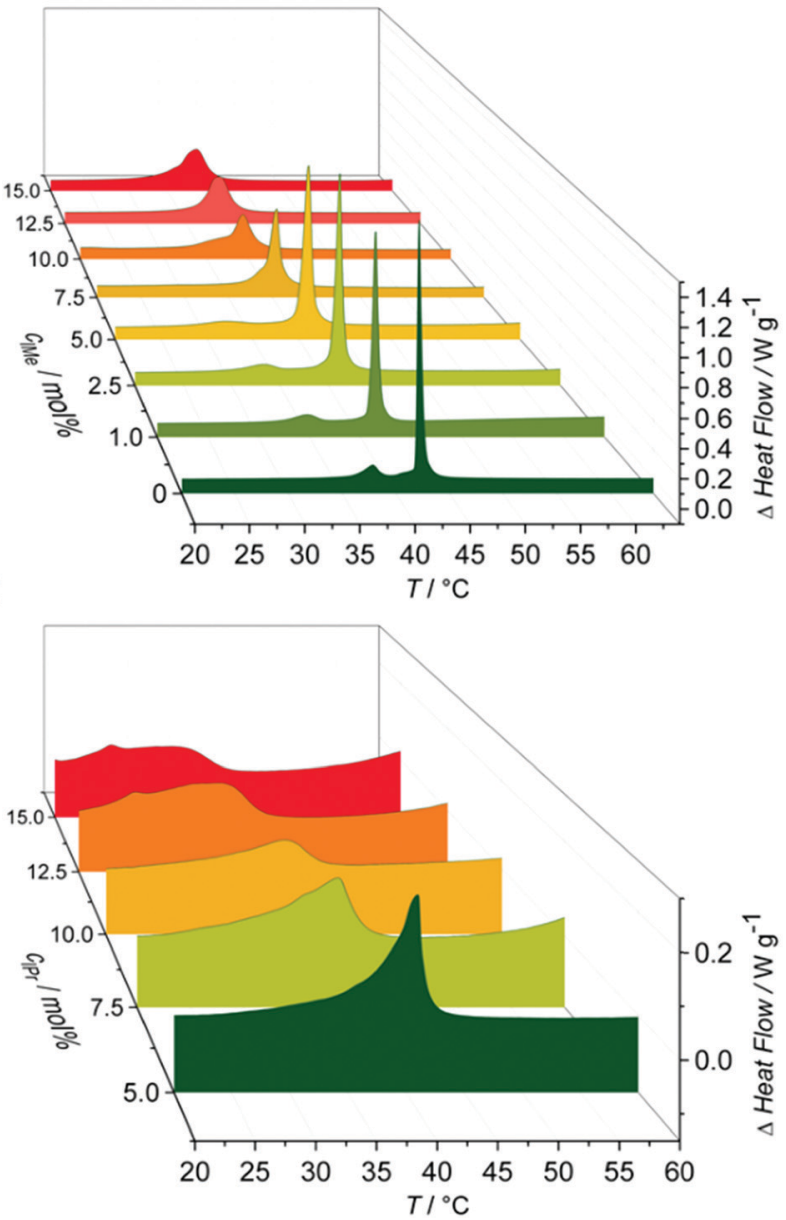

$\mathrm{B}$
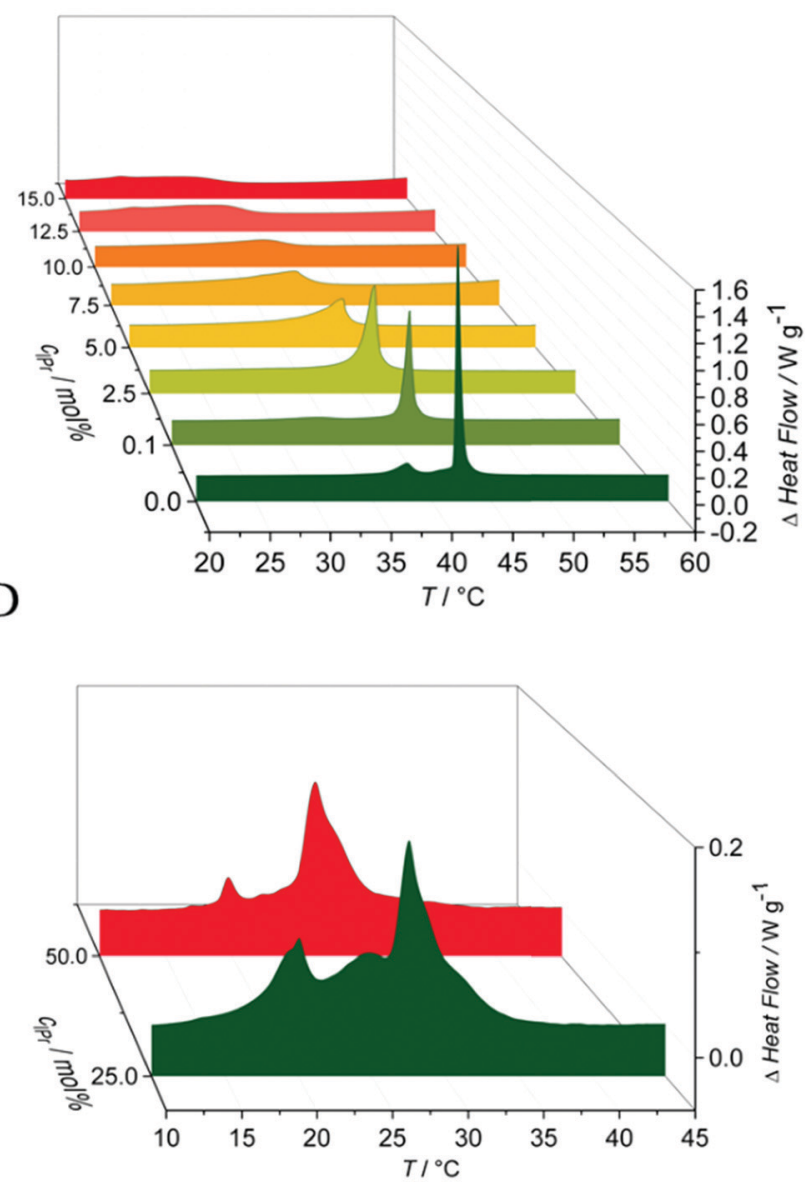

Fig. 5 DSC thermograms of pure DPPC vesicles upon addition of (A) $C_{11} I M e$ and (B)-(D) $C_{11} I P r$ at different concentrations. In (A), the disappearance of the pretransition peak is observed at about $5.0 \mathrm{~mol} \% \mathrm{C}_{11} \mathrm{IMe}$, and leads to a distinct peak broadening at higher concentrations, indicating phase coexistence regions. In (B), the insertion of $\mathbf{C}_{11} I \mathrm{Pr}$ leads to a disappearance of the pretransition already at 1 mol\%. Further increase of the fraction of $\mathbf{C}_{11} \mathrm{IPr}$ leads to a pronounced peak broadening, as better visualized in (C). Insertion of $25 \mathrm{~mol} \%$ and 50 mol\% $\mathrm{C}_{11}$ IPr leads to the formation of two separate transition peaks, indicating the formation of additional distinct $\mathbf{C}_{11} 1 \mathrm{Pr}$ domains or micellar structures.

A

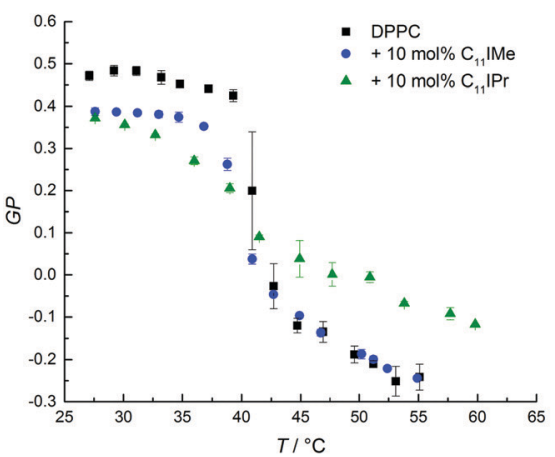

B

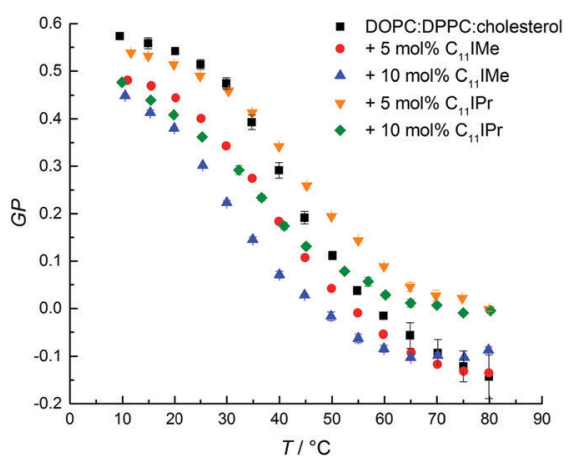

$\mathrm{C}$

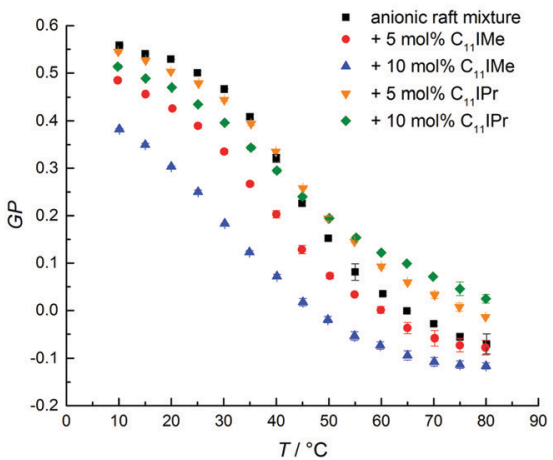

Fig. 6 Representative results of the Laurdan GP data for (A) DPPC with $10 \mathrm{~mol} \% \mathbf{C}_{11} I \mathrm{Me}$ and $10 \mathrm{~mol} \% \mathrm{C}_{11} \mathrm{IPr}$, (B) DPPC : DOPC : cholesterol with $10 \mathrm{~mol} \%$ $\mathrm{C}_{11} \mathrm{IMe}$ and 10 mol\% $\mathrm{C}_{11} \mathrm{IPr}$ and $(\mathrm{C})$ the anionic five component raft mixture with 10 mol\% $\mathrm{C}_{11} \mathrm{IMe}$ and $10 \mathrm{~mol} \% \mathrm{C}_{11} \mathrm{IPr}$.

The GP-curves seen in Fig. 6A show a sigmoidal-like progression and the sample containing neat DPPC displays almost constant $G P$-values up to the main transition temperature at about
41.9 ${ }^{\circ} \mathrm{C}$, where the transition from the gel phase to the fluid $\mathrm{L}_{\alpha}$ phase sets in. The acyl chains in the fluid $\mathrm{L}_{\alpha}$ phase have a high conformational disorder, consisting of a significant number of 
gauche conformers and kinks. In the fluid phase, water can diffuse easily into the upper bilayer region, leading to an increase in the intensity of the red-shifted $490 \mathrm{~nm}$ fluorescence band and a corresponding decrease of the $G P$-value, reaching even negative values. The main phase transition temperature determined is in good agreement with known literature values. ${ }^{35,46}$ Below $T_{\mathrm{m}}$, in the gel phases, all-trans conformations of the acyl chains prevail, and the lateral and rotational diffusion of the lipids is restricted, rendering the local environment of Laurdan less polar, which leads to a fluorescence emission maximum located at about $440 \mathrm{~nm}$ and a corresponding increase of the $G P$, reaching $G P$-values around 0.5 in densely packed, solid-ordered gel phases.

As shown in Fig. 6, the addition of $\mathbf{C}_{\mathbf{1 1}}$ IMe and $\mathbf{C}_{\mathbf{1 1}}$ IPr has similar effects on the GP-curves for all membrane systems. In the gel phase, the $G P$-values decrease upon the addition of the imidazolium salts in a concentration-dependent manner, and the chain-melting transitions are broadened. Thereby, $\mathbf{C}_{\mathbf{1 1}} \mathbf{I M e}$ has a more pronounced effect than $\mathbf{C}_{\mathbf{1 1}} \mathbf{I P r}$, whereas $\mathbf{C}_{\mathbf{1 1}}$ IPr shows a more distinct effect on the $G P$-values at temperatures above $T_{\mathrm{m}}$. I.e., the overall lipid order is markedly increased compared to the pure lipid system. The behavior of $\mathbf{C}_{\mathbf{1 1}} \mathbf{I P r}$ is reminiscent of that cholesterol is imposing on DPPC, showing a decrease of lipid order in the gel phase and the opposite in the fluid-like phase. ${ }^{47}$ Here, the bulky headgroup of $\mathbf{C}_{\mathbf{1 1}} \mathbf{I P r}$ is expected to prevent close packing of DPPC molecules in the gel phase, but could, probably owing to intermolecular $\pi-\pi$ interactions of their headgroups, lead to an increase of the overall lipid order parameter above $T_{\mathrm{m}}$. Conversely, $\mathbf{C}_{\mathbf{1 1}} \mathbf{I M e}$ shows no significant effect on the GP-values at higher temperatures. Further, no concentration dependence is observed. Both imidazolium salts lower the main transition temperature of DPPC, the effect of $\mathbf{C}_{\mathbf{1 1}}$ IPr being more pronounced $\left(T_{\mathrm{m}}(\mathrm{DPPC})=41.9 \pm 0.1{ }^{\circ} \mathrm{C}, T_{\mathrm{m}}\left(\mathrm{DPPC}+10 \mathrm{~mol} \% \mathbf{C}_{\mathbf{1 1}} \mathbf{I M e}\right)=40.8 \pm\right.$ $\left.0.1{ }^{\circ} \mathrm{C}, T_{\mathrm{m}}\left(\mathrm{DPPC}+10 \mathrm{~mol} \% \mathbf{C}_{\mathbf{1 1}} \mathrm{IPr}\right)=38.4 \pm 0.1{ }^{\circ} \mathrm{C}\right)$. The small differences in observed $T_{\mathrm{m}}$ values between the different methods applied are due to the different sensitivity of the methods. Because of the broad phase transition regions of the 3- and 5-component lipid systems, a determination of phase transition temperatures of the phase coexistence regions is difficult, only general trends of the lipids' overall lipid order can be discussed. The general trends upon addition of the imidazolium salts are similar to those of the simple lipid systems, such as the effect of $\mathbf{C}_{\mathbf{1 1}}$ IPr on ordered and fluid lipid phases.

\subsection{Zeta potential measurements}

To obtain also information about the effect of the imidazolium salt insertion on the surface charge density of the lipid membranes, we performed measurements of the zeta potential, s, i.e. the electric potential at the layer that separates the hydrodynamically immobile solution layer at the lipid interface from the bulk medium, which in turn depends on the insertion of the (charged) lipidated imidazolium salts into the lipid bilayers. Since the model membrane systems are neutral or anionic, the insertion of the cationic imidazolium salts $\mathbf{C}_{\mathbf{1 1}} \mathrm{IMe}$ and $\mathbf{C}_{\mathbf{1 1}}$ IPr are expected to increase the zeta potential or even change its sign, similar to the effects of cation adsorption at lipid interfaces. ${ }^{36,48}$

Fig. 7A and B show the results for the neutral zwitterionic DPPC membrane and for the anionic, five-component model membrane, respectively. Addition of $10 \mathrm{~mol} \% \mathbf{C}_{\mathbf{1 1}} \mathbf{I M e}$ to the DPPC membrane results in a significant increase of the zeta potential, from $0 \mathrm{mV}$ in DPPC up to about $+12 \mathrm{mV}$ in the presence of $10 \mathrm{~mol} \% \mathbf{C}_{\mathbf{1 1}} \mathbf{I M e}$, indicating efficient integration into the lipid DPPC bilayer. The insertion of $10 \mathrm{~mol} \% \mathbf{C}_{\mathbf{1 1}} \mathbf{I P r}$ results also in a distinct increase of the zeta potential, however up to about $6 \mathrm{mV}$ only. This also indicates uptake by the lipid membrane. The smaller potential may be explained by a lower insertion efficiency of the $\mathbf{C}_{\mathbf{1 1}} \mathbf{I P r}$, or, more likely, may result from the shielding of the positive charge of the $\mathbf{C}_{\mathbf{1 1}}$ IPr's headgroup by its surrounding bulky residues (see Fig. 1). The zeta potential of the pure, anionic membrane is about $-16 \mathrm{mV}$. Insertion of the $\mathbf{C}_{\mathbf{1 1}} \mathbf{I M e}$ salt increases the potential by about $12 \mathrm{mV}$, to about $-4 \mathrm{mV}$, which is a value that is similar to the increase observed for the DPPC membrane. Addition of $10 \mathrm{~mol} \%$ of the $\mathbf{C}_{\mathbf{1 1}}$ IPr salt, however, resulted in the aggregation of the sample after extrusion, thus preventing carrying out these measurements. Assuming a similar potential increase compared to the neutral membrane as in the $\mathbf{C}_{\mathbf{1 1}}$ IMe case, we would expect a potential value
A

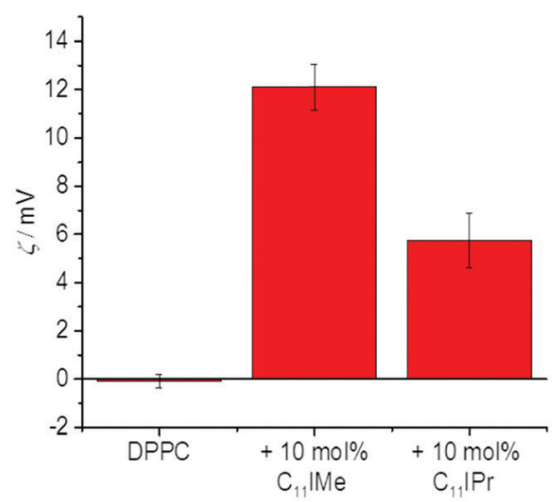

B

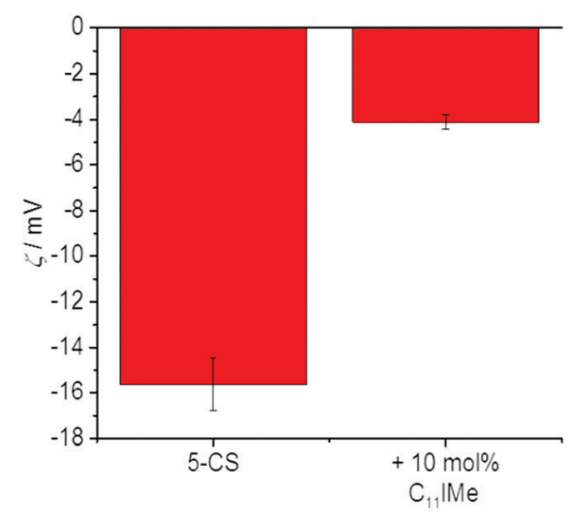

Fig. 7 Zeta potential measurements of (A) the pure, neutral (zwitterionic) DPPC membrane and (B) the anionic, five-component raft mixture before and after the addition of $10 \mathrm{~mol} \% \mathbf{C}_{11}$ IMe and $10 \mathrm{~mol} \% \mathbf{C}_{11}$ IPr. Insertion of the imidazolium salts results in a significant increase of the zeta potential, more pronounced for the $\mathbf{C}_{11} \mid \mathrm{Me}$ salt, indicating better and/or more homogeneous insertion into the membrane relative to the $\mathbf{C}_{11} I \mathrm{Pr}$ salt. 
around $-10 \mathrm{mV}$, i.e. a value close to zero. Since high zeta potentials of $\varsigma>20 \mathrm{mV}$ are required to stabilize vesicles effectively against aggregation, potentials near $0 \mathrm{mV}$ are prone to lead to spontaneous aggregation due to the lack of electrostatic repulsion, as probably observed here. ${ }^{46}$ Another aspect, which may increase the aggregation propensity of the $\mathbf{C}_{\mathbf{1 1}} \mathbf{I P r}$ is $\pi-\pi$-stacking caused by the interaction of the diisopropylphenyl-groups. ${ }^{49}$ Both effects combined may lead to the aggregation of the $\mathbf{C}_{\mathbf{1 1}}$ IPr containing vesicles. To explore this effect in more detail, we engaged in fluorescence as well as atomic force microscopic studies, which are discussed below.

\subsection{Fluorescence microscopy studies of $\mathrm{C}_{11} \mathrm{IMe}$ and $\mathrm{C}_{11} \mathrm{IPr}$ containing membranes}

To capture the effects of the imidazolium salts on the mesoscopic structure and phase behavior of lipid vesicles in more detail, we performed confocal fluorescence microscopy studies. To this end, we prepared giant unilamellar vesicles (GUVs)

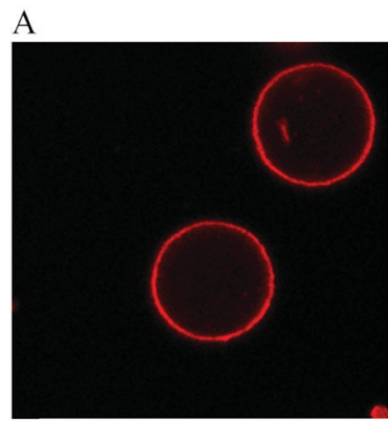

DPPC
B

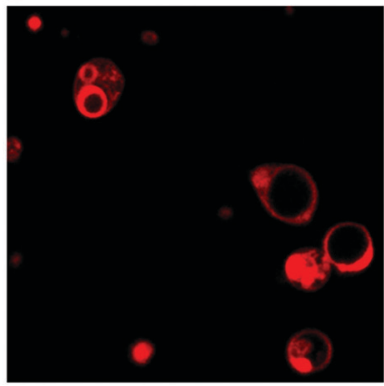

DPPC: $\mathrm{C}_{11} \mathrm{IPr}$

$(9: 1)$

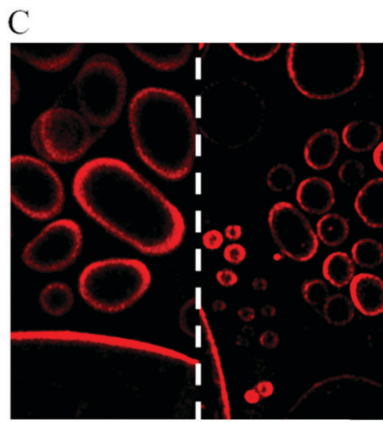

DPPC: $\mathrm{C}_{11} \mathrm{IPr}$

$(1: 1)$

Fig. 8 Confocal fluorescence microscopy cross-section images of the impact of the $\mathrm{C}_{11} \mathrm{IPr}$ salt on the topology of pure DPPC membranes. The red fluorescence originates from the fluorescence marker $N$-Rh-DHPE.

A

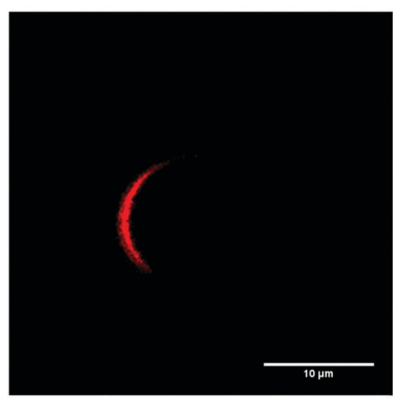

DPPC:DOPC:cholesterol $(2: 1: 1)$
B

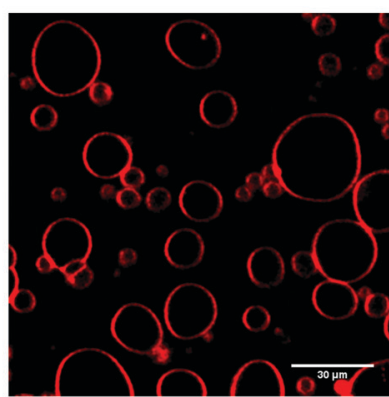

DPPC:DOPC:cholesterol:C $\mathrm{C}_{11} \mathrm{IMe}$ (9:5:5:1)

$\mathrm{D}$

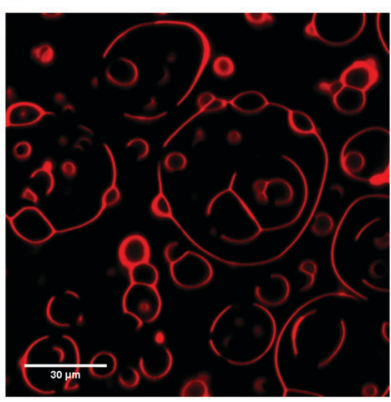

DPPC:DOPC:cholesterol: $\mathrm{C}_{11} \mathrm{IPr}$ (9:5:5:1)
$\mathrm{C}$

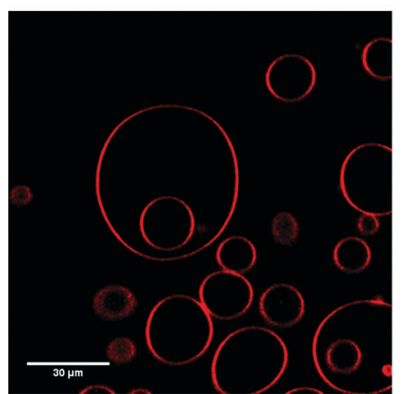

DPPC:DOPC: cholesterol: $\mathrm{C}_{11} \mathrm{IMe}$ $(8: 5: 5: 2)$

$\mathrm{E}$

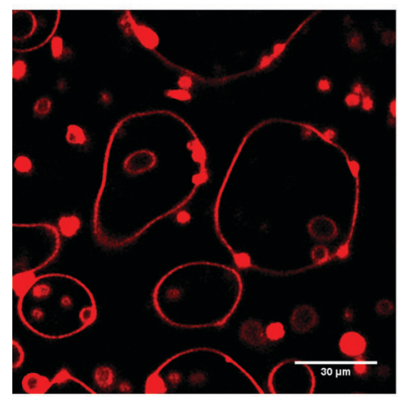

DPPC:DOPC:cholesterol: $\mathrm{C}_{11} \mathrm{IPr}$ $(8: 5: 5: 2)$

Fig. 9 Representative confocal fluorescence microscopy cross-section images of the impact of the $\mathbf{C}_{11} I \mathbf{M e}$ and $\mathbf{C}_{11} I \mathrm{Pr}$ salt on the structure of the neutral three component membrane consisting of DPPC : DOPC : cholesterol $(2: 1: 1)$ at $25^{\circ} \mathrm{C}$. The red fluorescence originates from the fluorescence marker N-Rh-DHPE. 
of pure DPPC vesicles, of the neutral three-component DPPC: DOPC:cholesterol mixture as well as of the anionic fivecomponent DPPC:DPPG:DOPC:DOPG : cholesterol lipid mixture. The lipid membrane was marked by the fluorescent probe $\mathrm{N}$-RhDHPE, which preferably partitions into the liquid-disordered phase and therefore marks the fluid domains of the membrane in case of $\mathrm{l}_{\mathrm{o}}-\mathrm{l}_{\mathrm{d}}$ phase coexistence. Data on the interaction of the $\mathbf{C}_{\mathbf{1 1}}$ IMe in pure DPPC vesicles have already been described, and in this case strongly fluidized and disordered DPPC liposomes were observed. ${ }^{26}$ Fig. 8 shows the marked topological changes upon inclusion of the $\mathbf{C}_{\mathbf{1 1}}$ IPr salt into the DPPC membrane. GUVs of pure DPPC vesicles display a spherical shape (A). A molar ratio of 9:1 of DPPC : $\mathbf{C}_{\mathbf{1 1}}$ IPr results in inclusions and protrusions (B), and the shape of GUVs with a lipid ratio of $1: 1$ for DPPC: $\mathbf{C}_{\mathbf{1 1}}$ IPr results in many floppy oval-shaped vesicles as well as small vesicles or detached mixed micelles or other small non-lamellar aggregate structures (C).

The impact of both imidazolium salts on the neutral three component membrane is shown in Fig. 9. Image A depicts the results for the neat DPPC: DOPC: cholesterol (2:1:1) system, which shows $l_{o}-l_{d}$ phase coexistence. The red fluorescence indicates the location of fluid $l_{d}$ phase. Image $B$ depicts the images for the 9:5:5:1 DPPC: DOPC: cholesterol: $\mathbf{C}_{\mathbf{1 1}}$ IMe mixture, which show small and large vesicles. Phase separation only occurred at low temperatures below $5{ }^{\circ} \mathrm{C}$ while the three-component mixture lacking the imidazolium salt still phase separates at higher temperatures of $50-60{ }^{\circ} \mathrm{C}$, indicating a highly fluidizing effect of the imidazolium salt on the membrane structure. The scale bar represents $30 \mu \mathrm{m}$, revealing a distinct growth in vesicle size upon insertion of the salt. Image $\mathrm{C}$ shows the images for the 8:5:5:2 DPPC:DOPC: cholesterol: $\mathbf{C}_{\mathbf{1 1}}$ IMe membrane, where no phase segregation and thermal phase transition was observed anymore. The vesicles differ in size from small to very large distorted (the scale bar represents $30 \mu \mathrm{m}$ ). Image D depicts the insertion of $\mathbf{C}_{\mathbf{1 1}}$ IPr into the membrane at a ratio of $9: 5: 5: 1$ DPPC: DOPC : cholesterol: $\mathbf{C}_{\mathbf{1 1}}$ IPr. Phase segregation is still partially visible at $55{ }^{\circ} \mathrm{C}$, and the vesicle sizes vary from small to very large. Insertion of the $\mathbf{C}_{\mathbf{1 1}}$ IPr salt also results in an aggregation of vesicles, indicating interactions between the inserted molecules of different vesicles. Increase of the mole fraction of the $\mathbf{C}_{\mathbf{1 1}}$ IPr salt up to 8: $5: 5: 2$ DPPC : DOPC : cholesterol : $\mathbf{C}_{\mathbf{1 1}}$ IPr leads to large, distorted vesicles next to small spherical ones and detached, possibly micellar particles. Phase transitions still occur at temperatures of about $25{ }^{\circ} \mathrm{C}$.

Fig. 10 displays the impact of both imidazolium salts on the morphology of the anionic five component DPPC:DPPG : DOPC: DOPG : cholesterol raft mixture at different mole fractions of the imidazolium salts. Image A depicts the neat $45: 5: 20: 5: 25$ DPPC: DPPG:DOPC:DOPG: cholesterol GUVs at room temperature,

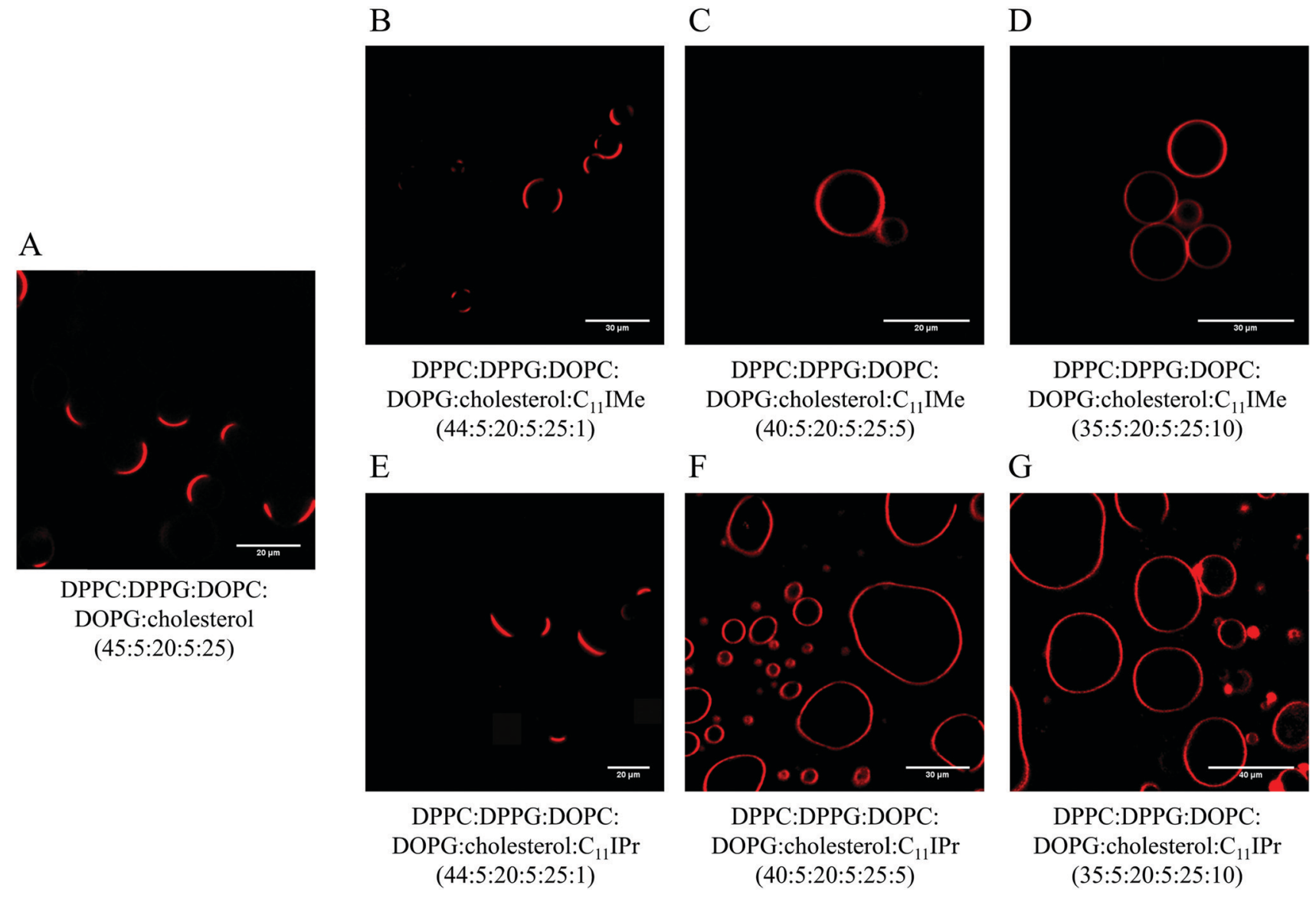

Fig. 10 Representative confocal fluorescence microscopy cross-section images of the impact of the $\mathbf{C}_{11} I \mathbf{M e}$ and $\mathbf{C}_{\mathbf{1 1}} \mathbf{I P r}$ salts on the structure of the anionic five component raft mixture membrane consisting of DPPC: DPPG : DOPC : DOPG : cholesterol $(45: 5: 20: 5: 25)$ at $25{ }^{\circ} \mathrm{C}$. 
clearly showing $\mathrm{l}_{\mathrm{O}}-\mathrm{l}_{\mathrm{d}}$ phase separation. Inclusion of the $\mathbf{C}_{\mathbf{1 1}} \mathbf{I M e}$ salt to $44: 5: 20: 5: 25: 1$ DPPC:DPPG:DOPC:DOPG : cholesterol: $\mathbf{C}_{11}$ IMe mixture did not show significant changes to the neat membrane system. Increase of the mole fraction of the imidazolium salt to $40: 5: 20: 5: 25: 5$ DPPC:DPPG:DOPC:DOPG : cholesterol: $\mathbf{C}_{11} \mathrm{IMe}$, however, results already in an increased vesicle size and phase separation only below $7{ }^{\circ} \mathrm{C}$ instead of about $75{ }^{\circ} \mathrm{C}$ for the neat membrane mixture. Thus, the highly fluidizing effect of the $\mathbf{C}_{\mathbf{1 1}} \mathbf{I M e}$ salt that was seen for the neutral membrane is also observed by the anionic raft mixture. Further increase to $35: 5: 20: 5: 25: 10$ DPPC: DPPG : DOPC: DOPG : cholesterol: $\mathbf{C}_{\mathbf{1 1}}$ IMe leads to the disappearance of the phase separation and to the formation of small, membrane attached particles, possibly mixed micellar structures.

Inclusion of the $\mathbf{C}_{\mathbf{1 1}}$ IPr salt to the anionic five component raft mixture at a ratio of $44: 5: 20: 5: 25: 1$ DPPC: DPPG : DOPC:
DOPG : cholesterol: $\mathbf{C}_{\mathbf{1 1}}$ IPr does not lead to remarkable changes in the vesicle structure compared to the neat membrane. As shown in image $F$, the increase of the $\mathbf{C}_{\mathbf{1 1}} \mathbf{I P r}$ salt fraction to $40: 5: 20: 5: 25: 5$ DPPC:DPPG:DOPC:DOPG: cholesterol : $\mathbf{C}_{11}$ IPr results in a reduced phase transition temperature of $7{ }^{\circ} \mathrm{C}$, similar to the $\mathbf{C}_{\mathbf{1 1}} \mathbf{I M e}$ system. Furthermore, small detached particles can be observed. The vesicle sizes are heterogeneous with large, deformed vesicles next to spherical, smaller ones. As depicted in image $\mathrm{G}$, further increase of the $\mathbf{C}_{\mathbf{1 1}}$ IPr salt fraction leads to very large, deformed GUVs with several small ones attached, possibly mixed micellar structures, and a drastic increase of the vesicle sizes compared to the neat membrane in image A. For the temperature dependent confocal microscopy images of the DOPC:DPPC:cholesterol and anionic five-component membrane systems, see the ESI. $\dagger$
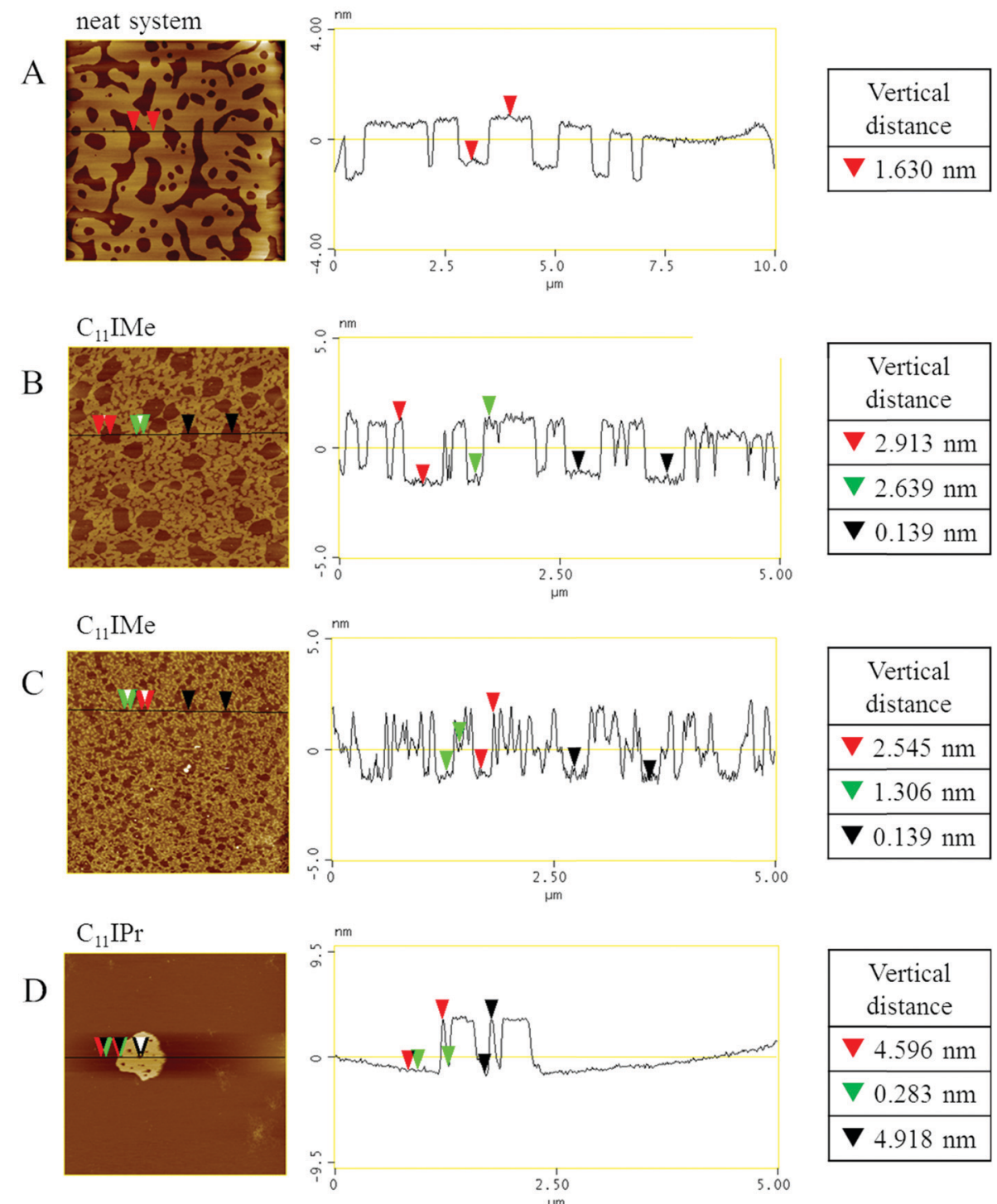

Fig. 11 Representative AFM images of the hydrated membranes with and without incorporated imidazolium salts. (A) Neat, neutral three-component lipid system. (B) Three-component lipid system +10 mol\% $\mathbf{C}_{11} \mathbf{I M e}$, measured directly after sample preparation. (C) Three-component lipid system + $10 \mathrm{~mol} \% \mathrm{C}_{11} \mathrm{IMe} 90 \mathrm{~min}$ after sample preparation. (D) Three-component lipid system $+10 \mathrm{~mol} \% \mathrm{C}_{11} \mathrm{IPr}$. The scan sizes of these images are $10 \mu \mathrm{m} \times 10 \mu \mathrm{m}$ for the neat membrane and $5 \mu \mathrm{m} \times 5 \mu \mathrm{m}$ for the remaining images. 
Ostensible differences in the effectiveness of the ILs impact on the different lipid systems may be attributed to the fluorescence microscopy images that have been selected here. Clearly, however, the largest impact was seen for the DPPC:DPPG:DOPC:DOPG: cholesterol : $\mathbf{C}_{\mathbf{1 1}} \operatorname{IPr}(35: 5: 20: 5: 25: 10)$ system, where the vesicles increased significantly in size next to the changes of the other physico-chemical parameters discussed above.

\subsection{Atomic force microscopy (AFM)}

To gain information of the impact of the imidazolium salts on the structure and morphology of the membranes at the submicrometer scale, we employed also atomic force microscopy. To avoid any distortions on the membrane, the measurements were taken in a fluid AFM cell, so that the membrane structures can be investigated in their fully hydrated state. To this end, we prepared a bilayer of the neutral three component lipid system on an atomically flat mica surface and included $\mathbf{C}_{\mathbf{1 1}} \mathbf{I M e}$ as well as $\mathbf{C}_{\mathbf{1 1}}$ IPr salt at different concentrations in the lipid mixture. As shown in Fig. 11, the neat, neutral three-component lipid system shows distinct phase separation into liquid-ordered $\left(\mathrm{l}_{\mathrm{o}}\right.$, bright) and liquid-disordered $\left(\mathrm{l}_{\mathrm{d}}\right.$, dark) domains. The height difference of about $1.6 \mathrm{~nm}$ between those two phases is in good agreement with literature data. ${ }^{44,50,51}$ Insertion of the $\mathbf{C}_{\mathbf{1 1}}$ IMe salt into the lipid membrane and investigation of the bilayer thickness and height difference between phases directly after preparation shows a drastic decrease in the mean domain size and a dispersion of the liquid-disordered domains, likely due to a decrease of the line-tension at the domain boundaries of the two phases upon incorporation of the imidazolium salt. The height difference between the two phases of about $2.6 \mathrm{~nm}$ to $2.9 \mathrm{~nm}$ increased $1 \mathrm{~nm}$ with respect to the neat bilayer membrane. With time, e.g. $90 \mathrm{~min}$ after the preparation the three-component system with included $\mathbf{C}_{\mathbf{1 1}} \mathbf{I M e}$ salt (Fig. 11C), further dispersion of the phases is detected. Furthermore, three different levels of membrane heights can be determined here. The first level reaches about $2.5 \mathrm{~nm}$ from the liquid-disordered level similar to the height difference directly after preparation. The second level has a height difference of about $1.3 \mathrm{~nm}$ only, indicating formation of a more corrugated lateral structure and formation of segregated $\mathbf{C}_{\mathbf{1 1}}$ IMe clusters of smaller heights.

The insertion of the $\mathbf{C}_{\mathbf{1 1}} \mathbf{I P r}$ salt into the neutral membrane shows a strong accumulation of the salt in the membrane, only. Image D shows the highly aggregated cluster with membrane height differences of about $4.5 \mathrm{~nm}$. Attempts to prepare AFM samples of the inserted cationic imidazolium salts into the heterogeneous, anionic five component membranes failed. Insertion of $\mathbf{C}_{\mathbf{1 1}}$ IMe and $\mathbf{C}_{\mathbf{1 1}}$ IPr into the membrane prevented the lipid system to form a stable bilayer on the mica surface. This behavior is in line with the results of the zeta potential measurements, where aggregation of the sample without forming stable unilamellar vesicles was observed as well.

\section{Conclusions}

Due to their high stability, large variability and easy possibility for modular synthesis, imidazolium salts have become attractive for many applications in material science and in biological systems in recent years. ${ }^{8,9,17}$ Since 2015, imidazolium salts bearing long alkyl chains in their backbone were established as lipid analogs, and, depending on the characteristics of their structure, different effects upon intercalation into phospholipid membranes have been reported..$^{21-23,25,26}$ Further, imidazolium salts have been shown to exhibit general cytotoxicity as well as significant anti-tumor activity.

Here, we examined the interaction of the 1,3-bis(2,6-diisopropylphenyl)-4,5-diundecylimidazolium bromide $\left(\mathbf{C}_{\mathbf{1 1}} \mathbf{I P r}\right)$ compound on the structure and physico-chemical properties of model biomembrane systems of different complexity, including also anionic heterogeneous lipid bilayers which mimic natural plasma membrane systems more closely. We compared the results obtained with those for the smaller headgroup variant 1,3-dimethyl-4,5-diundecylimidazolium iodide $\left(\mathbf{C}_{\mathbf{1 1}} \mathbf{I M e}\right)$ and studied the influence of these two lipid-based imidazolium salts at different concentrations on three different model biomembrane systems, i.e. DPPC, DPPC:DOPC: cholesterol and the anionic heterogeneous raft membrane DPPC:DPPG:DOPC:DOPG: cholesterol. We employed a variety of thermodynamic, spectroscopic and microscopic techniques to yield comprehensive insights into the effects of these lipidated imidazolium salts on lipid membranes, focusing in particular on their effect on the lateral organization of the lipid bilayers, which has not been addressed in detail before.

Both imidazolium salts lower the thermotropic gel-to-fluid phase transition temperature of phospholipid bilayers, hence reveal a marked fluidizing effect on lipid membranes. $\mathbf{C}_{\mathbf{1 1}} \mathbf{I P r}$ displays a more pronounced effect than $\mathbf{C}_{\mathbf{1 1}}$ IMe for all investigated bilayer systems, as expected from its larger headgroup (Fig. 1). ${ }^{23}$ The gel-to-gel pretransition of DPPC disappears already at low salt concentrations, indicating marked packing defects imposed by the two added imidazolium compounds. The surface zeta potential of the lipid membranes increases significantly for both imidazolium salts, demonstrating efficient insertion into the lipid bilayer systems. The incorporated lipidated imidazolium salts are even able to induce charge inversion in anionic membranes. This opens up possible applications for drug delivery and gene transfer, i.e. lipid vesicle-mediated transfer of negatively charged DNA or RNA.

Both lipid-like imidazolium salts lower the liquid-ordered to liquid-disordered phase transition temperature in heterogeneous membranes, and lead to a deterioration of the lateral domain structure of the membrane, i.e. the formation of a more corrugated lateral structure, most likely due to a decrease of line tension at the domain boundaries; this is to say, they act as linactants as their packing parameter does not fit the other lipids' shape. Such changes in the lateral organization of the more-component membranes will have severe consequences for membrane-assisted signaling and transport processes. Moreover, partitioning of the two lipidated imidazolium salts in the lipid vesicles leads to marked morphological changes of the GUVs at high concentrations, with $\mathbf{C}_{\mathbf{1 1}}$ IPr having a more pronounced effect on the shape of the GUVs than $\mathbf{C}_{\mathbf{1 1}} \mathbf{I M e}$. The AFM measurements reveal a strong segregation effect of both imidazolium salts in the anionic heterogeneous membrane, leading to a high positive charge 
density in these segregated areas. In comparison to the $\mathbf{C}_{\mathbf{1 1}} \mathbf{I M e}$, $\mathbf{C}_{\mathbf{1 1}}$ IPr segregates into larger domains, resulting in a significant increase of the membrane curvature. This effect on the membrane curvature was also seen in the fluorescence microscopic measurements of the two raft membrane mixtures by promoting enlarged and distorted vesicle shapes. Such effects of decreasing the bending rigidity and increasing the curvature of membranes are considered necessary for initiation of membrane fusion processes, again pointing to possible applications regarding drug or gene delivery. ${ }^{52,53}$

These results presented here clearly show that membrane interactions of 4,5-dialkylimidazolium salts strongly depend not only on the chain length, but also on the bulkiness of the headgroup region, and their interactions can lead, depending on their size, charge and chemical make-up, from a mere fluidizing effect up to marked changes in lateral membrane organization, lipid domain structure and vesicular shape. Hence, they seem to be highly suitable for biochemical and biotechnological engineering, with high potentials for antimicrobial activity (via membrane disruption and leakage), drug delivery (improving drug permeability) and gene transfer (increasing the fusogenicity of vesicles).

\section{Conflicts of interest}

There are no conflicts to declare.

\section{Acknowledgements}

Financial support from the DFG (German Research Foundation, GRK 2515/1) is gratefully acknowledged.

\section{References}

1 A. J. Arduengo, R. L. Harlow and M. Kline, J. Am. Chem. Soc., 1991, 113, 361-363.

2 M. N. Hopkinson, C. Richter, M. Schedler and F. Glorius, Nature, 2014, 510, 485-496.

3 C. Richter, K. Schaepe, F. Glorius and B. J. Ravoo, Chem. Commun., 2014, 50, 3204-3207.

4 F. E. Hahn and M. C. Jahnke, Angew. Chem., Int. Ed., 2008, 47, 3122-3172.

5 T. Dröge and F. Glorius, Angew. Chem., Int. Ed., 2010, 49, 6940-6952.

6 D. J. Nelson and S. P. Nolan, Chem. Soc. Rev., 2013, 42, 6723-6753.

7 G. C. Fortman and S. P. Nolan, Chem. Soc. Rev., 2011, 40, 5151-5169.

8 A. Bendetto, Biophys. Rev., 2017, 9, 309-320.

9 K. S. Egorova, E. G. Goordeev and V. P. Ananikov, Chem. Rev., 2017, 117, 7132-7189.

10 S. N. Riduan and Y. Zhang, Chem. Soc. Rev., 2013, 42, 9055-9070.

11 C. M. Mahalakshmi, M. Karthick, M. Shanmugam and V. Chidambaranathan, Pharma Chem., 2015, 7, 14-19.

12 K. Bakshi, S. Mitra, V. K. Sharma, M. S. K. Jayadev, V. G. Sakai, R. Mukhopadhyay, A. Gupta and S. K. Ghosh, Biochim. Biophys. Acta, Biomembr., 2020, 1862, 183103.
13 C. Samorì, D. Malferrari, P. Valbonesi, A. Montecavalli, F. Moretti, P. Galletti, G. Sartor, E. Tagliavini, E. Fabbri and A. Pasteris, Ecotoxicol. Environ. Saf., 2010, 73, 1456-1464.

14 K. Rawat and H. B. Bohidar, J. Phys. Chem. B, 2012, 116, 11065-11074.

15 E. B. Anderson and T. E. Long, Polymer, 2010, 51, 2447-2454.

16 L. Carson, P. K. W. Chau, M. J. Earle, M. A. Gilea, B. F. Gilmore, S. P. Gorman, M. T. McCann and K. R. Seddon, Green Chem., 2009, 11, 492-497.

17 D. Wang, H.-J. Galla and P. Drücker, Biophys. Rev., 2018, 10, 735-746.

18 A. Cornellas, L. Perez, F. Comelles, I. Ribosa, A. Manresa and M. T. Garcia, J. Colloid Interface Sci., 2011, 355, 164-171.

19 M. Tischer, G. Pradel, K. Ohlsen and U. Holzgrabe, ChemMedChem, 2012, 7, 22-31.

20 D. Demberelnyamba, K.-S. Kim, S. Choi, S.-Y. Park, H. Lee, C.-J. Kim and I.-D. Yoo, Bioorg. Med. Chem., 2004, 12, 853-857.

21 D. Wang, C. Richter, A. Rühling, S. Hüwel, F. Glorius and H.-J. Galla, Biochem. Biophys. Res. Commun., 2015, 467, 1033-1038.

22 D. Wang, C. Richter, A. Rühling, P. Drücker, D. Siegmund, N. Metzler-Nolte, F. Glorius and H.-J. Galla, Chem. - Eur. J., 2015, 21, 15123-15126.

23 A. Rühling, D. Wang, J. B. Ernst, S. Wulff, R. Honeker, C. Richter, A. Ferry, H.-J. Galla and F. Glorius, Chem. - Eur. J., 2017, 23, 5920-5924.

24 G. Bhattacharya, S. Mitra, P. Mandal, S. Dutta, R. P. Giri and S. K. Ghosh, Biophys. Rev., 2018, 10, 709-719.

25 P. Drücker, A. Rühling, D. Grill, D. Wang, A. Draeger, V. Gerke, F. Glorius and H.-J. Galla, Langmuir, 2017, 33, 1333-1342.

26 D. Wang, D. H. de Jong, A. Rühling, V. Lesch, K. Shimizu, S. Wulff, A. Heuer, F. Glorius and H.-J. Galla, Langmuir, 2016, 32, 12579-12592.

27 G. B. Díaz, A. M. Cortizo, M. E. Garciía and J. J. Gagliardino, Lipids, 1988, 23, 1125-1128.

28 G. B. Ansell, J. N. Hawthorne and R. M. C. Dawson, Form and function of phospholipids, Elsevier Scientific Pub. Co, Amsterdam, New York, 2nd completely rev. and enl., 1973.

29 S. Mitra, D. Ray, G. Bhattacharya, R. Gupta, D. Sen, V. K. Aswal and S. K. Ghosh, Eur. Biophys. J., 2019, 48, 119-129.

30 S. L. Veatch and S. L. Keller, Biophys. J., 2003, 85, 3074-3083.

31 K. Bacia, P. Schwille and T. Kurzchalia, Proc. Natl. Acad. Sci. U. S. A., 2005, 102, 3272-3277.

32 K. Simons and E. Ikonen, Nature, 1997, 387, 569-572.

33 H. A. Lucero and P. W. Robbins, Arch. Biochem. Biophys., 2004, 426, 208-224.

34 K. Weise, S. Kapoor, C. Denter, J. Nikolaus, N. Opitz, S. Koch, G. Triola, A. Herrmann, H. Waldmann and R. Winter, J. Am. Chem. Soc., 2011, 133, 880-887.

35 Phospholipid Handbook, ed. G. Cevc, CRC Press, Marcel Dekker, New York, 1993.

36 S. Bornemann, M. Herzog and R. Winter, Phys. Chem. Chem. Phys., 2019, 21, 5730-5743.

37 O. Reis, R. Winter and T. W. Zerda, Biochim. Biophys. Acta, Biomembr., 1996, 1279, 5-16. 
38 M. Herzog, L. Li, H.-J. Galla and R. Winter, Colloids Surf., B, 2019, 173, 327-334.

39 M. Herzog, M. Dwivedi, R. K. Harishchandra, A. Bilstein, H.-J. Galla and R. Winter, Colloids Surf., B, 2019, 178, 404-411.

40 A. Rühling, D. Wang, J. B. Ernst, S. Wulff, R. Honeker, C. Richter, A. Ferry, H.-J. Galla and F. Glorius, Chem. - Eur. J., 2017, 23, 5920-5924.

41 M. I. Angelova, S. Soléau, Ph. Méléard, F. Faucon and P. Bothorel, Preparation of giant vesicles by external AC electric fields. Kinetics and applications, in Trends in Colloid and Interface Science VI, ed. C. Helm, M. Lösche and H. Möhwald, Steinkopff, 1992, pp. 127-131.

42 S. Janosch, C. Nicolini, B. Ludolph, C. Peters, M. Völkert, T. L. Hazlet, E. Gratton, H. Waldmann and R. Winter, J. Am. Chem. Soc., 2004, 126, 7496-7503.

43 H. Stein, S. Spindler, N. Bonakdar, C. Wang and V. Sandoghdar, Front. Physiol., 2017, 8, 63-79.

44 K. Weise, G. Triola, L. Brunsveld, H. Waldmann and R. Winter, J. Am. Chem. Soc., 2009, 131, 1557-1564.
45 M. Pühse, C. Jeworrek and R. Winter, Chem. Phys. Lipids, 2008, 152, 57-63.

46 R. Winter, F. Noll and C. Czeslik, Methoden der Biophysikalischen Chemie, Vieweg + Teuber, Wiesbaden, 2nd edn, 2011.

47 F. M. Harris, K. B. Best and J. D. Bell, Biochim. Biophys. Acta, Biomembr., 2002, 1565, 123-128.

48 E. Chibowski and A. Szcześ, Adsorption, 2016, 22, 755-765.

49 S. Sao, I. Mukherjee, P. De and D. Chaudhuri, Chem. Commun., 2017, 53, 3994-3997.

50 S. Kapoor, A. Werkmüller, C. Denter, Y. Zhai, J. Markgraf, K. Weise, N. Opitz and R. Winter, Biochim. Biophys. Acta, Biomembr., 2011, 1808, 1187-1195.

51 H. A. Rinia, M. M. E. Snel, J. P. J. M. van der Eerden and B. de Kruijff, FEBS Lett., 2001, 501, 92-96.

52 L. V. Chernomordik and M. M. Kozlov, Nat. Struct. Mol. Biol., 2008, 15, 675-683.

53 I. Koltover, T. Salditt, J. O. Rädler and C. R. Safinya, Science, 1998, 281, 78-81. 